\title{
Primary Umbilical Endometriosis: Report of a Rare Case
}

\author{
Dilip K. Bhowmik ${ }^{1^{*}}$ \\ Rezwana Mirza \\ Amzad Hossain ${ }^{2}$
}

'Department of Obstetrics and Gynaecology Sylhet MAG Osmani Medical College

Sylhet, Bangladesh.

${ }^{2}$ Department of Pathology

Sylhet MAG Osmani Medical College

Sylhet, Bangladesh

\section{*Correspondence to:}

\section{Dr. Dilip K. Bhowmik}

8/1 (1 st floor) Munsipara R/A

Sylhet 3100, Bangladesh.

Mobile: +8801711349775

E-mail: dbhowmiksomc@gmail.com

w w w.c mos h m c j.org

\begin{abstract}
Endometriosis is the commonest gynaecological problem; it affects 7 to $10 \%$ women in reproductive age group. Commonest site being the pelvic organs, extrapelvic presentation in almost all parts of the body have been reported. However umbilical endometriosis has been reported in more than one hundred cases. It was first described by Villar in 1886. Umbilical endometriosis accounts for only $0.5 \%$ to $1 \%$ of all endometriosis cases. Most of the reported cases occurred in the scar following gynaecological and obstetrical surgery. A few cases of primary umbilical endometriosis have been reported. We report such a rare case of primary umbilical endometriosis and the diagnosis was done by FNAC \& the patient underwent wide local excision.
\end{abstract}

Key words: Endometriosis; umbilical; cutaneous; primary.

\section{INTRODUCTION}

Endometriosis was first described by Rokitansky in 1860 and was defined as the presence of proliferation of endometrium outside the uterine cavity, commonest site being the pelvis ${ }^{1}$. It is a common gynecological condition that affects up to $22 \%$ of all women, $8-15 \%$ of reproductive age and $6 \%$ premenopausal women ${ }^{2-3}$. Endometriosis commonly found anywhere in the pelvic cavity: on the ovaries, fallopian tube, uterosacral ligaments, pouch of Douglus ${ }^{4}$. Extragenital endometriosis is less common, but has been described in almost every area of the female body including the bowel, bladder, lungs, brain, umbilicus, liver and surgical scars ${ }^{5}$. Umbilical endometriosis represents $0.5-1 \%$ of all cases of endometriosis, which was first described by Villar in $1886^{6}$. However umbilical endometriosis has been reported in more than hundred cases. The majority of the reported cases occurred in obstetrical \& gynecologically induced abdominal or pelvic scars including hysterectomy, episiotomy, caeserian section \& laparoscopy ${ }^{7}$. Few cases of primary umbilical endometriosis have been reported ${ }^{2,3,7,8}$. We report one such a rare case of primary umbilical endometriosis.

\section{CASE REPORT}

A 42 years old woman, para 4-2, admitted with a slowly growing umbilical nodule of 3 years duration. It was associated with cyclical localized pain and bleeding during periods of menstruation (Fig 1). Her menstrual cycle was regular with average flow and duration. She has no history of pelvic pain or abdominal pain. There was no history of dysmenorrhoea and dyspareunia. No history of abdominal and pelvic surgery was noted. Her bladder and bowel habit was normal. She did not practice any contraceptive method. She used to take some analgesics during pain, but no history of medication for this complains. On examination, the nodule was about $4 \mathrm{~cm} \mathrm{x} 2 \mathrm{~cm} \mathrm{x}$ $1.5 \mathrm{~cm}$ in size, with involving umbilicus, soft to firm in consistency, brown to black in colour, was tender and non reducible. Per vaginal examinations revealed- her vulva, vagina \& cervix were healthy. Uterus was normal in size and anteverted. Fornices were free and not tender. No lump or nodule felt. Ultrasound of abdomen showed no other significant contributory finding. FNAC of the lesion showed endometriosis. The patient underwent a wide local excision of the nodule (Fig 2) with umbilical reconstruction with prior counseling regarding risk of recurrence. Histopathology confirmed the diagnosis of umbilical endometriosis (Fig 3). Her postoperative period was uneventful. The patient was asymptomatic at 6 months of follow up but nevertheless warned the risk of recurrence. 

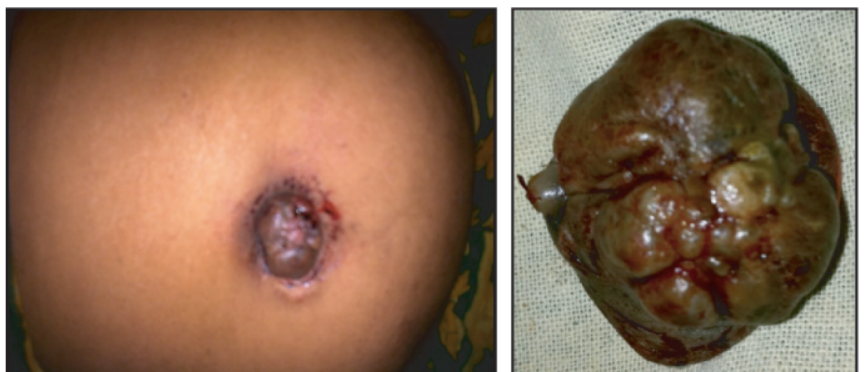

Figure 1: Photograph showing menstruating primary umbilical endometriosis.

Figure 2: Dissected umbilical nodule with endometriosis measuring about $4 \mathrm{~cm} \times 2 \mathrm{~cm} \times 1.5 \mathrm{~cm}$.

\section{DISCUSSION}

Primary (spontaneous) umbilical endometriosis was first described by Villar in 1886 which represents about $0.5-1 \%$ of all cases of extra genital endometriosis. Endometriosis involving the abdominal wall is termed as cutaneous endometriosis which is commonly with surgical scars ${ }^{9-10}$. Clinically the lesion appears as firm nodule varying in size from a few millimeters to centimeters. Cyclical pain with palpable mass is the commonest presenting symptom. The pathogenesis of endometriosis has been a topic under constant debate during the last decade. Sampson hypothesized in 1920s that endometriosis resulted from retrograde menstruation through the fallopian tube into the pelvis ${ }^{11}$. However, several other theories exists for the development of endometriosis including coelomic metaplasia, direct spread, iatrogenic dissemination, lymphatic \& hematogenous spread ${ }^{2}$. Real mechanism still remains a mystery. Theory of lymphatic and hematogenous transplantation is favoured in the case of umbilical endometriosis with co existing pelvic endometriosis. However, it is believed that the disease might arise through metaplasia of urachal remnants in case of isolated umbilical endometriosis ${ }^{3,10}$. The possible theory proposed by $\mathrm{Yn}$ et al is that the umbilicus, as a physiological scar, appears to become an early focus for ectopic endometrial implantation. In one systemic literature review, the commonest symptoms in patients with umbilical endometriosis are swelling $(90.9 \%)$, pain $(81.5 \%)$ and bleeding (49.2\%). Most umbilical endometriosis presented with brown in color followed by blue, purple, black and $\mathrm{red}^{10}$. A few series have reported intermittent bleeding from the nodule. Our patient, having history of slowly growing umbilical nodule with cyclical pain during menstruation and also bleeding from the mass, gives a picture characteristic of cutaneous endometriosis. But the characteristic symptom is not always present and clinical diagnosis of the umbilical endometriosis may be difficult. So when a mass developed at umbilicus, the possibility of endometrioma must be kept in mind although there may be other differential diagnosis include granuloma, umbilical hernia, lipoma, abscess, cyst, melanoma, primary or metastatic adenocarcinoma (sister joshep's nodule) ${ }^{2}$. Differential diagnoses also include primary cancer of umbilicus (basal cell \& squamous cell carcinoma), melanoma \& paget's disease $^{4}$. The cytological features of cutaneous \& sub cutaneous endometriosis are related to cyclic hormonal changes ${ }^{2,7}$. The cytological smears generally shows cellular with epithelial \& stromal fragments admixed with hemorrhage \& hemosiderin laden macrophages ${ }^{7}$.
Cytological findings of FNAC of our patient revealed a moderately cellular smear containing strips \& sheets of endometrial epithelial cells. Endometrial stromal cells were scanty. No cellular atypia or atypical mitosis was noted. Specimen also included degenerated red blood cells with hemosiderin laden macrophage. A cytodiagnosis of umbilical endometriosis was made. The imaging modalities are non specific but are useful in determining the extent of disease and planning of operative resection especially of large and recurrent lesions ${ }^{1}$. Thus FNAC is a fast and accurate method to make the diagnosis before surgery avoiding errors in approach of the umbilical nodule ${ }^{12}$

The treatment of umbilical endometriosis consists of medical and surgical approach. But hormone therapy may lead to incomplete regression of umbilical lesion. Therefore, surgery is the only choice of treatment ${ }^{6}$. Preoperative hormone therapy may be used in patients with large mass of umbilical endometriosis to reduce its size but subsequent surgical excision remains the treatment of choice. In our case, the lesion was excised and umbilicus was reconstructed. Histology confirmed the diagnosis. Although simultaneous laparoscopy has been recommended for pelvic endometriosis, this was not done because our patient was asymptomatic. Although local recurrence is uncommon, the patient has been warned of the risk of scar endometriosis and of recurrence. After six months follow up, the patient remains asymptomatic and there was no recurrence of the lesion.

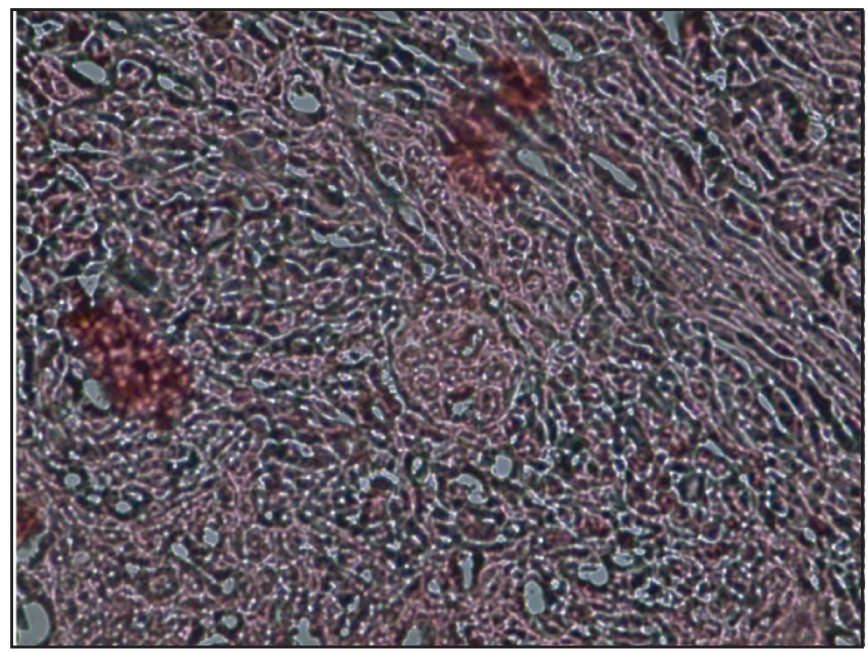

Figure 3: Histopathology of umbilical nodule showing numerous tubular glands lined by columnar epithelium (endometrial glands) with the presence of fibro-vascular tissue in the background.

\section{CONCLUSION}

Umbilical endometriosis is a rare presentation of extra pelvic endometriosis. It should be considered as a differential diagnosis in an umbilical lesion of woman of reproductive age. Clinical features and MRI \& FNAC are useful diagnostic tools and surgical excision is the standard treatment option.

\section{DISCLOSURE}

All the authors declared no competing interest. 
REFERENCES

1. Thapa A, Kumar A, Gupta S. Abdominal wall endometriosis: Report of A case \& How much we know about it. Internet J. Surg 2007, 9:(S).

2. Catalina-Fernández 1, López-Presad, Sáenz-Santamaria J. Fine needle aspiration- cytology in cutaneous \& subcutaneous endometriosis. Actacytol 2007;51:380-4

3. Agarwal A, Fond YF. Cutaneous endometriosis. Singapore Med J 2008;49:704-9

4. D'ttooghe TM, Hill JA. Endometriosis in:Berek JS, Adashi EY, Hillard PA, editors. Novak's gynecology. $13^{\text {th }}$ ed. Lippincott Williams \& Wilkins; 2002:887-914.

5. Markham SM, Carpenter SE, Rock JA. Extrapelvic endometriosis obstet Gynecol Chin North Am. 1989; 16: 193-219

6. Latcher JW. Endometriosis of the umbilicus. Am. J Obstet Gynecol. 1953; 66: 161-168 [Pub Med]

7. Medeiros FC, Cavalcante DI, Medeiros MA, Elenterio J. Jr. Fine needle aspiration cytology of scar endometriosis: study of seven cases \& literature review. Diagn Cytopathol 2011; 39: 18-21

8. Pathan ZA, Dinesh W, Rao R. Scar endometriosis. J Cytol 2010; 27: 106-8.

9. Khaled A, Hammami H, Fazaa B, Zemani R, Ben Jilani S, Kamoun MR. Primary umbilical endometriosis; a rare variant of extragenital endometriosis. Pathologice 2008; 100: 473-5.

10. Victory R, Diamondon P, Johns DA. Villar's nodule, a case report with systemic literature review of endometriosis externa of the J Minim Invagine Gynecol 2007; 14: 23-32.

11. Arara S, Iger VK, Mathur SR. Cytological diagnosis of peritoneal endometriosis. J Cytol 2010; 27: 77-78.

12. Fernandes H, Maria NJ, Pailwor K, Kini R. Primary umbilical endometriosis- Diagnosed by fine needle aspiration. J Cytol 2011; 28: 214-6. 